Revta brasil. Bot., São Paulo,V.24, n.4 (suplemento), p.553-560, dez. 2001

\title{
Variabilidade intra-específica em cinco populações de Oncidium varicosum Lindl. (Orchidaceae - Oncidiinae) em Minas Gerais
}

\author{
DARUSKA CAVALCANTE CARDIM ${ }^{1}$, LUCIANA APARECIDA CARLINI-GARCIA $^{1}$, MATEUS \\ MONDIN $^{1}$, MARINA MARTINS ${ }^{1}$, ELIZABETH ANN VEASEY $^{1,2}$ e AKIHIKO ANDO ${ }^{1}$
}

(recebido: 4 de setembro de 2000; aceito: 27 de junho de 2001)

\begin{abstract}
Intraspecific variability in five populations of Oncidium varicosum Lindl. (Orchidaceae - Oncidiinae) in Minas Gerais). Five populations of Oncidium varicosum Lindl. (Orchidaceae - Oncidiinae) collected in Minas Gerais at Alfenas (AL), Estiva (ES), Pouso Alegre (PA), Pedra do Coração (PC) and Pedra Branca (PB), were examined with the aim of studying the intraspecific genetic variability. Twenty-two floral characters were measured from vouchers, using univariate and multivariate statistical methods (canonical discriminant analysis - CDA and cluster analysis using Mahalanobis distance). Significant differences $(p<0,05)$ among populations, by the $F$ test, were observed for 13 characters. The first canonical axis separated population AL from PB and PC. The PA individuals are smaller than those of PC, which are smaller than those of PB. AL overlapped PA, ES and $\mathrm{PC}$ by the first axis, but was completely separated from PA and almost totally from the other populations according to the second canonical axis, for presenting characteristics such as longer and thicker columns, longer and narrower lateral sepals and shorter ovaries. According to the cluster analysis, AL diverged again from the other populations. Populations ES, PC and PB are closer to each other, followed by PA. The pattern of genetic variability observed does not seem to be related to different latitudes. However, it seems to be associated to longitudinal differences, although this single factor may not be enough to explain this pattern.
\end{abstract}

RESUMO - (Variabilidade intra-específica em cinco populações de Oncidium varicosum Lindl. (Orchidaceae - Oncidiinae) em Minas Gerais). Com o objetivo de estudar a variabilidade genética intra-específica em Oncidium varicosum Lindl. (Orchidaceae - Oncidiinae), foram avaliadas cinco populações de Minas Gerais, coletadas em Alfenas (AL), Estiva (ES), Pedra do Coração (PC), Pedra Branca (PB) e Pouso Alegre (PA). Foram considerados 22 caracteres florais, medidos a partir de "vouchers" (fichas florais), empregando-se métodos estatísticos univariados e multivariados (análise discriminante canônica - CDA e análise de agrupamento a partir de distância de Mahalanobis). Houve diferença significativa $(p<0,05)$ entre populações, pelo teste $F$, para 13 caracteres. O primeiro eixo canônico separou a população PA de PB e de PC. Os indivíduos de PA são menores que os de PC, que são menores que os de PB. AL se sobrepôs a PA, ES e PC pelo primeiro eixo, mas se separou completamente de PA e quase que totalmente das demais populações de acordo com o segundo eixo canônico, por apresentar características como colunas compridas e espessas, sépalas laterais compridas e estreitas e ovários mais curtos. De acordo com a análise de agrupamento, novamente a população AL divergiu das demais. Observou-se que as populações ES, PC e PB são mais próximas entre si, seguidas da população de PA. O padrão de variabilidade genética observado não parece estar relacionado a diferenças de latitude. Entretanto, parece estar associado a diferenças de longitude, embora este único fator pode não ser suficiente para explicá-lo.

Key words - Orchidaceae, Oncidium varicosum, morphometric analysis, genetic variability, multivariate analysis.

\section{Introdução}

Dentro da família Orchidaceae, a subtribo Oncidiinae é uma das mais importante do Novo Mundo, ocorrendo na América Tropical. Embora compreendendo 77 gêneros, metade das 1232 espécies dessa subtribo estão distribuídas entre os

1. Universidade de São Paulo, Escola Superior de Agricultura "Luiz de Queiroz", Departamento de Genética, Caixa Postal 83, 13400-970 Piracicaba, SP.

2. Autor para correspondência: eaveasey@carpa.ciagri.usp.br gêneros Oncidium e Odontoglossum (Dressler 1981, 1993). A polinização é realizada por insetos (Dressler 1993).

Por se tratar de uma das maiores famílias vegetais e por ter origem filogenética recente (Terciário Inferior), Brieger (1971) baseou-se na evolução filogenética e distribuição geográfica de espécies de orquídeas, assim como em espécies das famílias Araceae e Cactaceae, para tirar conclusões sobre ocorrências geológicas durante períodos geológicos anteriores, em regiões tropicais. Brieger $(1961,1976)$ utilizou a família Orchidaceae para estudar evolução nos trópicos, já que são muito freqüentes nessa região e apresentam grande 
diversidade. Decorre daí a importância de estudos com essa família.

Oncidium varicosum Lindl. ocorre principalmente em florestas úmidas da região semitropical dos Estados de Minas Gerais, São Paulo e Goiás. Apresenta flores dispostas em panícula (McQueen \& McQueen 1993). Características desta espécie são os lóbulos laterais do labelo arredondados e a calosidade do labelo com sua protuberância central em forma de unicórnio (Pabst 1972). As sépalas e pétalas são subsimilares, inconspícuas, notavelmente clavadas ou constritas na base. As sépalas laterais são mais curtas que o labelo. Os discos do labelo apresentam números variáveis de tubérculos. O rostelo é curto (Garay \& Stacy 1974).

Muitos trabalhos de revisão taxonômica do gênero Oncidium são encontrados na literatura (Pabst 1972, Garay \& Stacy 1974, Dressler 1981, 1993). Entretanto, pouco se sabe sobre a variabilidade genética de suas populações. Métodos morfométricos podem ser usados com sucesso na inferência da variabilidade genética de espécies vegetais (Loos 1993, Brysting \& Elven 2000).
O objetivo deste trabalho foi estudar o padrão de variabilidade genética em cinco populações de Oncidium varicosum de Minas Gerais, a partir da análise morfométrica de caracteres florais.

\section{Material e métodos}

Foram estudadas cinco populações de Oncidium varicosum Lindl. do Estado de Minas Gerais. As populações amostradas pertencem às localidades de Alfenas (AL), Pouso Alegre (PA), Estiva (ES), Pedra do Coração (PC) e Pedra Branca (PB), no município de Pocinho do Rio Verde, em diferentes altitudes (figura 1, tabela 1). As amostras, coletadas aleatoriamente em seus locais de origem, possuem diferentes tamanhos, respeitando a abundância das populações na natureza (tabela1). As plantas coletadas foram levadas para $o$ Orquidário pertencente ao Departamento de Genética da Escola Superior de Agricultura "Luiz de Queiroz" (ESALQ/USP), onde foram mantidas em condições ambientais uniformes por vários anos, o que garante que a variação apresentada por elas seja predominantemente de origem genética. Por ocasião do florescimento, foram feitas fichas florais ("vouchers"), que estão depositadas no herbário ESA (ESALQ/USP), a partir das quais foram medidos vinte e dois caracteres florais (tabela 2). Para cada caráter, considerou-se a média de três a seis flores por planta, conforme a disponibilidade de "vouchers". Todos os caracteres foram medidos em milímetros.

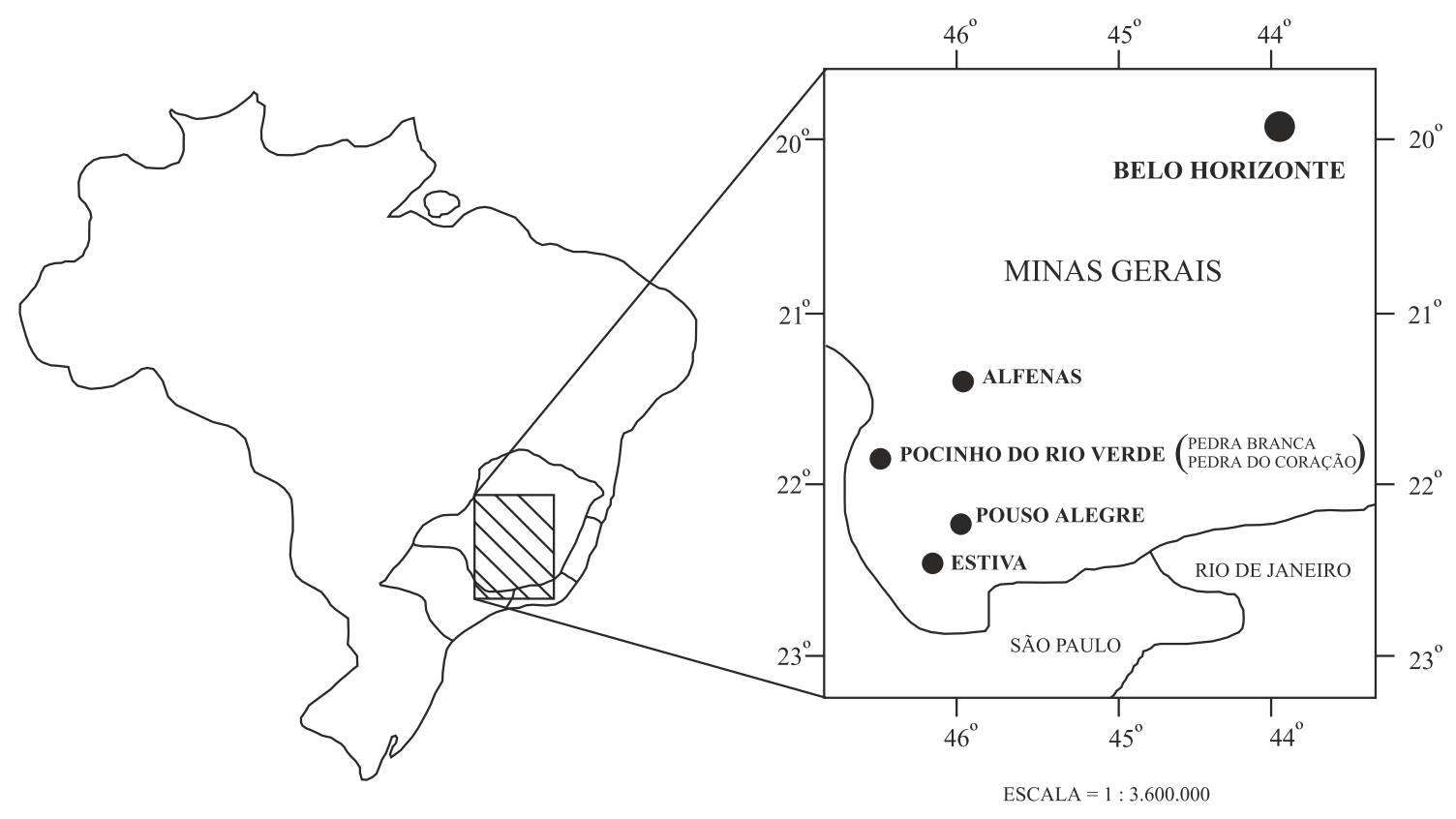

Figura 1. Mapa do Estado de Minas Gerais com os locais de coleta das populações avaliadas. 
Revta brasil. Bot., São Paulo,V.24, n.4 (suplemento), p.553-560, dez. 2001

Tabela 1. Populações de Oncidium varicosum, com respectivos tamanhos amostrais e altitudes dos locais de coleta.

\begin{tabular}{lcc}
\hline \multicolumn{1}{c}{ População } & Tamanho amostral & Altitude $(\mathrm{m})$ \\
\hline Alfenas (AL) & 5 & 750 \\
Pouso Alegre (PA) & 10 & 800 \\
Estiva (ES) & 12 & 800 \\
Pedra do Coração (PC) & 26 & 1400 \\
Pedra Branca (PB) & 8 & 1500 \\
\hline
\end{tabular}

Avaliaram-se apenas caracteres florais. O emprego de caracteres florais em estudos morfométricos e taxonômicos é vantajoso, pois, devido à especificidade entre flor e agente polinizador, a variabilidade presente nesses caracteres é constituída quase que exclusivamente por variabilidade genética, sendo pouco influenciados pelo ambiente. Procedimento semelhante foi usado por Knyasev et al. (2000), que com base na análise morfométrica de caracteres florais estudou as relações taxonômicas entre três espécies de Cypripedium (Orchidaceae).

Foram feitas análises de variância univariadas e teste $\mathrm{F}$ para cada caráter isoladamente. Para os caracteres cujo teste $\mathrm{F}$ foi significativo, foi realizado teste t para contrastes entre as médias das populações. Análises de correlação de Pearson entre pares de caracteres foram realizadas, uma vez que a presença de correlações significativas justifica o uso de análises multivariadas. O nível mínimo de significância considerado em todas essas análises foi $5 \%$.

Distâncias Generalizadas de Mahalanobis $\left(\mathrm{D}^{2}\right)$ foram calculadas entre pares de populações. Esta distância foi escolhida por considerar a variação intra-específica e as correlações entre as variáveis. Com base nestas distâncias, foram obtidos os fenogramas entre as populações. Utilizaramse três métodos de agrupamento aglomerativos: método de ligação simples, que é o único método que delineia grupos elipsoidais (Andenberg 1973); UPGMA, amplamente difundido na literatura e WPGMA, devido ao desbalanceamento entre as populações. $\mathrm{O}$ coeficiente de correlação cofenética foi calculado para cada método de agrupamento usado. Quanto maior o valor deste coeficiente, melhor a representação da matriz original de distâncias (Sneath \& Sokal 1973).

A análise discriminante canônica foi empregada para verificar como as populações relacionam-se entre si. Esta análise foi utilizada por considerar as correlações residuais existentes entre as médias das observações, o que se traduz numa vantagem em relação aos componentes principais. $\mathrm{Na}$ análise de variáveis canônicas, o princípio de conglomeração baseia-se na distância de Mahalanobis. Outra vantagem do método é que, mesmo que o número de variáveis não seja menor que o número de indivíduos dentro de cada população e que a multinormalidade não esteja presente, a análise

Tabela 2. Relação dos 22 caracteres florais avaliados em cinco populações de Oncidium varicosum e siglas correspondentes.

\begin{tabular}{ll}
\hline Siglas dos caracteres & \multicolumn{1}{c}{ Caracteres } \\
\hline CTL & Comprimento total do labelo \\
LTL & Largura total do labelo \\
CCL & Comprimento central do labelo \\
CE & Comprimento esquerdo do labelo \\
CD & Comprimento direito do labelo \\
CEA & Comprimento esquerdo do labelo incluindo o apêndice superior \\
CDA & Comprimento direito do labelo incluindo o apêndice superior \\
CLS & Comprimento do apêndice superior \\
CL2 & Comprimento do labelo a 25\% da largura total do labelo \\
CL5 & Comprimento do labelo a 50\% da largura total do labelo \\
CL7 & Comprimento do labelo a $75 \%$ da largura total do labelo \\
CP & Comprimento da pétala \\
LP & Largura da pétala \\
CSD & Comprimento da sépala dorsal \\
LSD & Largura da sépala dorsal \\
CSL & Comprimento da sépala lateral \\
LSL & Largura da sépala lateral \\
CPE & Comprimento do pedúnculo \\
CCOL & Comprimento da coluna \\
LCOL & Largura da coluna \\
ECOL & Espessura da coluna \\
OV & Comprimento do ovário \\
\hline
\end{tabular}


Tabela 3. Análise de variância e teste $F$ para os 22 caracteres $^{1}$ avaliados em cinco populações de Oncidium varicosum.

\begin{tabular}{|c|c|c|c|c|c|c|c|c|c|c|c|c|}
\hline \multirow[t]{2}{*}{ F.V. } & \multirow[t]{2}{*}{ G.L. } & \multicolumn{11}{|c|}{ QUADRADOS MÉDIOS } \\
\hline & & CTL & LTL & $\mathrm{CCL}$ & $\mathrm{CE}$ & $\mathrm{CD}$ & CEA & $\mathrm{CDA}$ & CLS & CL2 & CL5 & CL7 \\
\hline Populações & 4 & $25,79^{*}$ & $84,09^{* *}$ & $7,25^{\mathrm{ns}}$ & $64,39^{* *}$ & $61,73^{* *}$ & $23,83^{*}$ & $18,47^{\mathrm{ns}}$ & $1,47^{\mathrm{ns}}$ & $41,58^{* *}$ & $94,46^{* *}$ & $49,90^{* *}$ \\
\hline Resíduo & 56 & 9,06 & 15,96 & 4,44 & 10,41 & 11,85 & 8,42 & 10,03 & 2,45 & 7,52 & 12,55 & 8,61 \\
\hline \multirow[t]{2}{*}{ F.V. } & G.L. & \multicolumn{11}{|c|}{ QUADRADOS MÉDIOS } \\
\hline & & $\mathrm{CP}$ & LP & CSD & LSD & CSL & LSL & CPE & $\mathrm{CCOL}$ & $\mathrm{LCO}$ & ECOL & OV \\
\hline Populações & 4 & $2,04^{\mathrm{ns}}$ & $0,92^{\mathrm{ns}}$ & $2,78^{*}$ & $0,11^{\mathrm{ns}}$ & $3,90^{*}$ & $0,49^{\mathrm{ns}}$ & $97,31^{* *}$ & $0,48^{\mathrm{ns}}$ & $1,41^{*}$ & $0,89^{\mathrm{ns}}$ & $8,48^{* *}$ \\
\hline Resíduo & 56 & 0,94 & 0,47 & 1,04 & 0,24 & 1,07 & 0,35 & 11,32 & 0,21 & 0,53 & 0,37 & 1,37 \\
\hline
\end{tabular}

discriminante canônica pode ser aplicada de maneira exploratória, produzindo resultados gráficos bastante úteis (Manly 1994). Diversos estudos têm mostrado a utilidade do emprego desses métodos multivariados em Orchidaceae como, por exemplo, DuPuy et al. (1985), Resende (1991), Dufrêne et al. (1991), van den Berg (1996), Carlini (1996), Brysting \& Elven (2000) e Knyasev et al. (2000).

\section{Resultados e Discussão}

Observaram-se diferenças significativas $(p<$ $0,05)$ entre populações, a partir da análise de variância e teste $F$, para os caracteres CTL, LTL, CE, CD, CEA, CL2, CL5, CL7, CSD, CSL, CPE, LCOL e OV (tabela 3 ). As médias e desviospadrões de todos os caracteres, bem como o teste $\mathrm{t}$ para contrastes entre as médias das populações para os caracteres que apresentaram diferenças significativas pelo teste $\mathrm{F}$, encontram-se na tabela 4. A alta correlação observada entre vários pares de caracteres (tabela 5) justifica a realização de análises multivariadas (Manly 1994).

Pela análise discriminante canônica verificou-se que o primeiro eixo canônico, que representa 55,37\% da variação, separou as populações $\mathrm{PA}$ e $\mathrm{PB}$, bem como as populações PA e PC. Ao observar conjuntamente as figuras 2 e 3 , nota-se que há um gradiente de tamanho, sendo que as flores dos indivíduos de PA são menores que as de PC, que por sua vez são menores que as de $\mathrm{PB}$. A população PB caracteriza-se principalmente por apresentar flores com ovários e pedúnculos mais compridos, labelos maiores e sépalas laterais mais estreitas em relação às flores das demais populações. A população $\mathrm{AL}$ se sobrepõe às populações PA, ES e PC pelo primeiro eixo mas, de acordo com o segundo eixo canônico, separa-se completamente de PA e quase que totalmente das demais populações (figura 2). A população AL caracteriza-se por apresentar flores com colunas compridas e espessas, sépalas laterais compridas e estreitas, grande CL7 e ovários mais curtos (figuras 2, 3). Esses resultados são concordantes com aqueles apresentados na tabela 4 . O segundo eixo

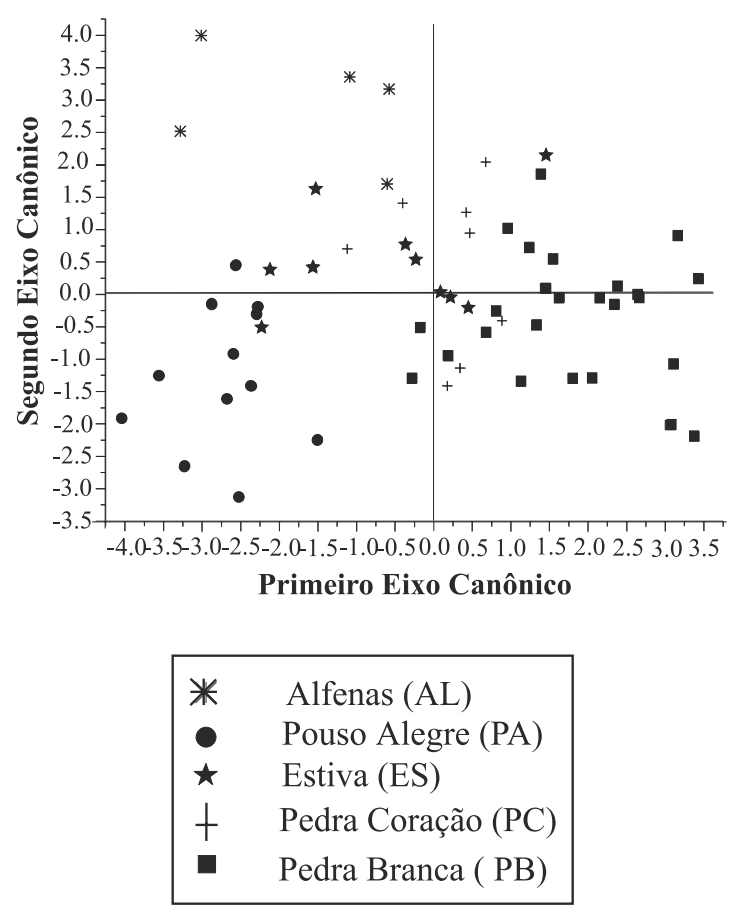

Figura 2. Escores provenientes da análise discriminante canônica entre cinco populações de Oncidium varicosum, considerando o primeiro e o segundo eixos canônicos. 
canônico representa $20,65 \%$ da variação observada. Somados, o primeiro e o segundo eixos representam $76,02 \%$ desta variação.

A partir das distâncias de Mahalanonis (tabela 6), observa-se que as populações AL e PB são as mais distantes entre si, em concordância com os dois eixos da análise discriminante canônica (figura 2), onde se observa a ocorrência de sobreposição de apenas um indivíduo pelo segundo eixo canônico. A população $\mathrm{AL}$ é a mais divergente entre todas, apresentando maiores distâncias em relação as demais, seguida pelas populações PA e PB (tabela 6). Os três métodos de agrupamento foram concordantes, indicando que os grupos são bem definidos. Em função disso, apenas foi apresentado o fenograma resultante da análise de agrupamento realizada de acordo com o método UPGMA (figura 4). Os coeficientes de correlação cofenética obtidos pelos três métodos foram elevados $(83,88 \%$, $86,91 \%$ e $86,68 \%$ para ligação simples, UPGMA e WPGMA, respectivamente). Estes valores indicam que a matriz de distância foi pouco distorcida no processo de agrupamento.

Observou-se na análise de agrupamento, que as populações ES, $\mathrm{PC}$ e $\mathrm{PB}$ são as mais próximas entre si, formando um grupo (figura 4), o que é confirmado pela análise canônica (figura 2), onde se nota a sobreposição dessas três populações de acordo com os dois eixos. A população PA mostrouse intermediária a este grupo e à população $\mathrm{AL}$,

Tabela 4. Médias e desvios-padrão (entre parênteses) em mm, considerando 22 caracteres $^{1}$ em cinco populações de Oncidium $^{2}$ varicosum.

\begin{tabular}{|c|c|c|c|c|c|}
\hline Caráter & $\begin{array}{l}\text { Alfenas } \\
(\mathrm{AL})\end{array}$ & $\begin{array}{l}\text { Pouso Alegre } \\
\text { (PA) }\end{array}$ & $\begin{array}{c}\text { Estiva } \\
\text { (ES) }\end{array}$ & $\begin{array}{c}\text { Pedra do Coração } \\
\text { (PC) }\end{array}$ & $\begin{array}{l}\text { Pedra Branca } \\
\text { (PB) }\end{array}$ \\
\hline$\overline{\mathrm{CTL}}$ & $25,78(0,91) \mathrm{ab}^{2}$ & $25,17(3,69) b$ & $28,22(2,57) \mathrm{a}$ & $27,83(2,52) a b$ & $28,33(3,16) \mathrm{a}$ \\
\hline LTL & $28,00(2,45) \mathrm{b}$ & $27,55(5,00) b$ & $32,61(3,78) a$ & $31,00(3,63) a b$ & $33,22(3,86) \mathrm{a}$ \\
\hline CCL & $19,73(0,69)$ & $18,83(2,18)$ & $20,51(1,58)$ & $20,72(1,77)$ & $20,51(2,45)$ \\
\hline $\mathrm{CE}$ & $18,06(1,22) b$ & $18,99(4,21) b$ & $23,11(2,71) \mathrm{a}$ & $22,72(3,37) \mathrm{a}$ & $23,46(3,08) a$ \\
\hline $\mathrm{CD}$ & $18,27(1,21) b$ & $19,27(4,65) b$ & $23,21(2,67) \mathrm{a}$ & $22,82(3,44) \mathrm{a}$ & $23,64(3,31) \mathrm{a}$ \\
\hline CEA & $25,62(0,85) a b$ & $25,20(3,44) b$ & $28,32(2,42) \mathrm{a}$ & $28,08(2,69) \mathrm{a}$ & $28,01(3,07) \mathrm{a}$ \\
\hline CDA & $25,79(1,26)$ & $25,31(4,24)$ & $28,04(2,54)$ & $27,69(2,73)$ & $27,88(3,14)$ \\
\hline CLS & $15,14(1,94)$ & $14,99(1,15)$ & $15,83(1,81)$ & $15,39(1,00)$ & $15,69(1,69)$ \\
\hline CL2 & $26,41(2,18) b c$ & $25,41(2,16) \mathrm{c}$ & $28,71(2,92) a b$ & $28,28(2,61) a b$ & $29,65(3,01) \mathrm{a}$ \\
\hline CL5 & $27,77(2,46) b c$ & $26,84(3,13) \mathrm{c}$ & $32,53(3,83) a$ & $30,97(3,68) a b$ & $32,91(3,71) \mathrm{a}$ \\
\hline CL7 & $19,74(1,73) \mathrm{a}$ & $16,42(2,54) b$ & $21,76(2,81) \mathrm{a}$ & $19,95(3,63) \mathrm{a}$ & $20,76(3,07) \mathrm{a}$ \\
\hline $\mathrm{CP}$ & $9,67(0,91)$ & $9,06(1,17)$ & $9,32(0,98)$ & $10,09(0,84)$ & $9,87(0,91)$ \\
\hline LP & $4,23(0,85)$ & $4,39(0,87)$ & $4,52(0,52)$ & $5,06(1,07)$ & $4,33(0,43)$ \\
\hline CSD & $9,22(0,90) \mathrm{ab}$ & $8,65(1,22) b$ & $8,95(1,04) a b$ & $9,88(0,99) \mathrm{a}$ & $9,59(0,94) a b$ \\
\hline LSD & $4,45(0,51)$ & $4,58(0,63)$ & $4,55(0,50) \mathrm{a}$ & $4,79(0,40)$ & $4,62(0,42)$ \\
\hline CSL & $11,55(1,29) \mathrm{ab}$ & $10,57(1,15) \mathrm{c}$ & $11,32(1,02) b c$ & $12,33(0,81) \mathrm{a}$ & $11,49(1,00) a b c$ \\
\hline LSL & $4,08(0,27)$ & $4,61(1,04)$ & $4,42(0,45)$ & $4,38(0,48)$ & $4,18(0,42)$ \\
\hline CPE & $23,56(4,31) b c$ & $20,63(2,79) \mathrm{c}$ & $23,45(3,55) \mathrm{c}$ & $26,63(1,14) a b$ & $27,02(3,75) \mathrm{a}$ \\
\hline $\mathrm{CCOL}$ & $6,90(0,32)$ & $6,57(0,53)$ & $6,95(0,44)$ & $7,17(0,36)$ & $6,79(0,48)$ \\
\hline LCOL & $4,07(0,89) \mathrm{c}$ & $4,19(0,71) b c$ & $4,98(0,72) a$ & $4,79(0,85) a b$ & $4,33(0,66) a b c$ \\
\hline ECOL & $4,67(0,41)$ & $4,29(0,86)$ & $5,08(0,61)$ & $4,81(0,53)$ & $4,67(0,52)$ \\
\hline $\mathrm{OV}$ & $4,50(1,18) \mathrm{c}$ & $5,08(0,99) b c$ & $5,62(0,72) a b$ & $6,03(1,20) a b$ & $6,67(1,35) a$ \\
\hline
\end{tabular}

1. Caracteres descritos na tabela $2 .$.

2. Populações com a mesma letra não diferem significativamente $(p>0,05)$ entre si pelo teste $t$. 


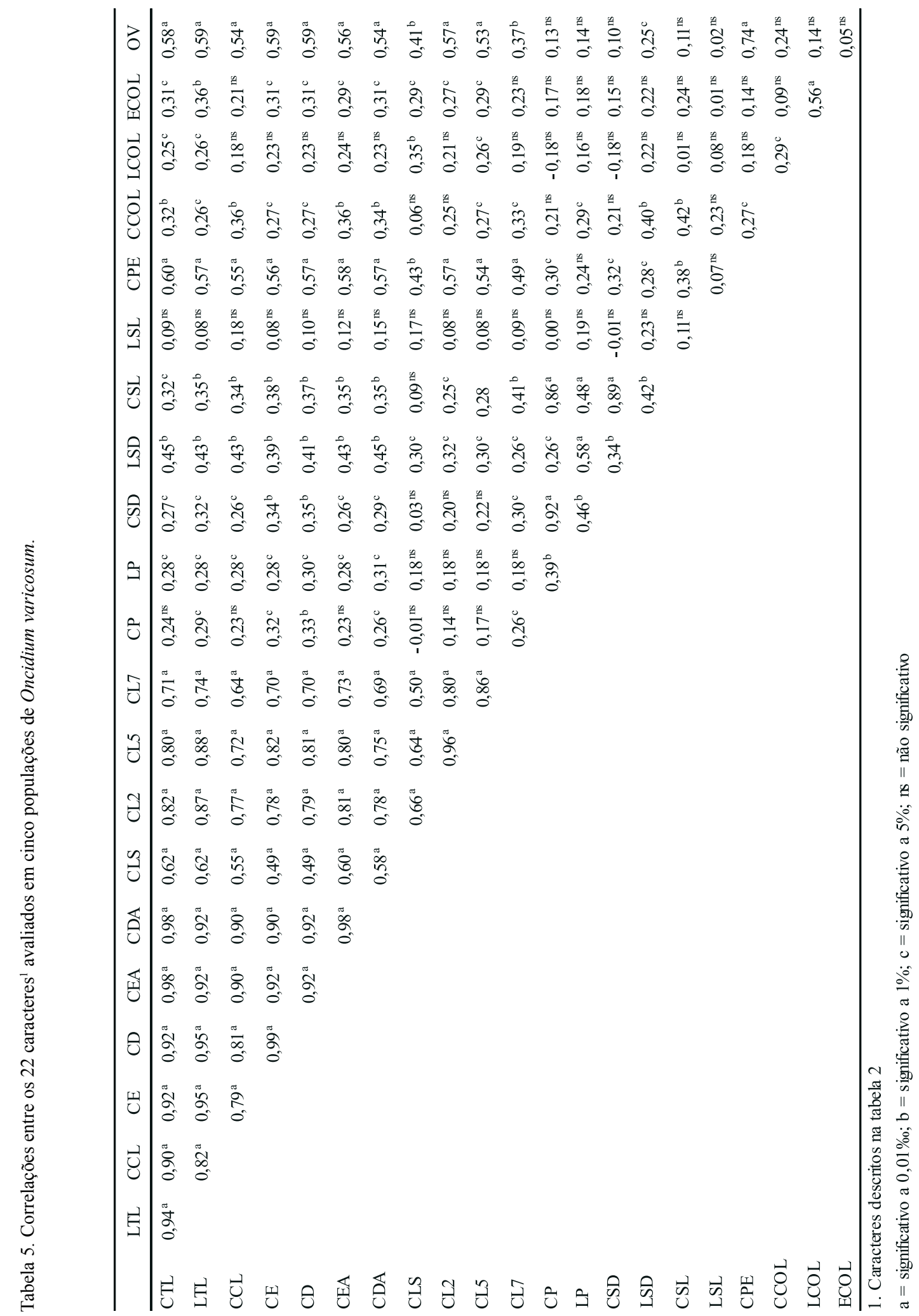




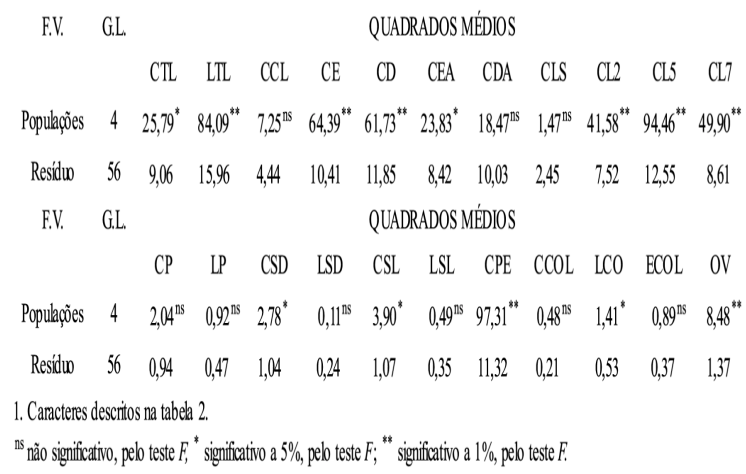

Figura 3. Contribuição das variáveis originais para o primeiro e segundo eixos canônicos entre cinco populações de Oncidium varicosum.

que é a mais divergente entre todas as populações (figura 4). Essas duas populações, AL e PA, encontram-se separadas na análise discriminante pelo segundo eixo canônico (figura 2). A partir dos resultados da análise de agrupamento, sugere-se que as cinco populações sejam classificadas em três grupos: o primeiro formado pelas populações ES, $\mathrm{PC}$ e $\mathrm{PB}$, que são mais próximas entre si, o segundo formado por PA e o terceiro por AL. Esses resultados são concordantes com aqueles obtidos pela análise discriminante canônica.

Knyasev et al. (2000), com base em análises morfométricas de peças florais, demonstraram que Cypripedium ventricosum Sw. é intermediária entre C. calceolus L. e C. macranthos Sw. Esses resultados foram concordantes com os obtidos a partir de análises isoenzimáticas. DuPuy et al. (1985) propuseram que Cymbidium wilsonii Rolfe

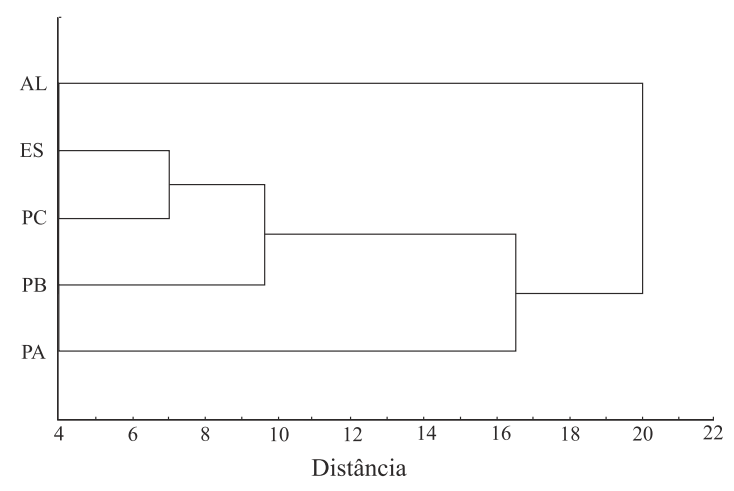

Figura 4. Fenograma obtido a partir da distância generalizada de Mahalanobis entre as populações Alfenas (AL), Estiva (ES), Pedra do Coração (PC), Pedra Branca (PB) e Pouso Alegre (PA) de Oncidium varicosum (Orchidaceae), pelo método UPGMA, considerando 22 caracteres florais.

e C. schroederi Rolfe tenham se originado por hibridação e ainda classificaram C. iansonii Rolfe como variedade de C. lowianum Rchb.f. Dufrêne et al. (1991) analisaram características quantitativas em trinta e cinco populações de Dactylorhiza maculata (L.) Soó. Consideraram também caracteres qualitativos, análises citológicas e observações ecológicas, e concluíram que características florais têm papel fundamental na distinção taxonômica.

No presente trabalho, ao estudar uma única espécie a partir de morfometria, foi possível detectar a existência de variabilidade entre populações geograficamente próximas. O padrão de variabilidade genética não parece estar associado a diferenças entre as altitudes e latitudes das populações estudadas. Populações semelhantes como ES e PC apresentaram grande diferença

Tabela 6. Distância Generalizada de Mahalanobis entre cinco

\begin{tabular}{lccccc}
\hline & $\begin{array}{c}\text { Alfenas } \\
(\mathrm{AL})\end{array}$ & $\begin{array}{c}\text { Estiva } \\
(\mathrm{ES})\end{array}$ & $\begin{array}{c}\text { Pouso Alegre } \\
(\mathrm{PA})\end{array}$ & $\begin{array}{c}\text { Pedra Branca } \\
(\mathrm{PB})\end{array}$ & $\begin{array}{c}\text { Pedra Coração } \\
(\mathrm{PC})\end{array}$ \\
\hline Alfenas (AL) & 0,00 & & & & \\
Estiva (ES) & 15,79 & 0,00 & & & \\
Pouso Alegre (PA) & 19,92 & 11,81 & 0,00 & 0,00 & 0,00 \\
Pedra Branca (PB) & 23,61 & 9,93 & 20,90 & 9,34 & \\
Pedra Coração (PC) & 20,70 & 7,02 & 16,83 & & \\
\hline
\end{tabular}


quanto à altitude, enquanto ES e AL, bastante divergentes entre si, foram coletadas praticamente na mesma altitude (tabela 1). Quanto à longitude, $\mathrm{PB}$ e PC são as populações que apresentam maiores longitudes, seguidas da população de ES. As populações de PA e AL estão localizadas em áreas de menor longitude, sendo mais afastadas das demais populações, fato que vem corroborar os resultados da análise de agrupamento. Entretanto, somente a variação de longitude talvez não seja suficiente para explicar o padrão de variabilidade encontrado nessas populações.

\section{Referências bibliográficas}

ANDERBERG, M.R. 1973. Clustering analysis for applications. Academic Press, London.

BRIEGER, F.G. 1961. A evolução filogenética nos trópicos. In Cadeira de Citologia e Genética Geral da Escola Superior de Agricultura "Luiz de Queiroz" da Universidade de São Paulo (F.G. Brieger et al., coords.). Atas do Primeiro Congresso Sul-Americano de Genética, p.154-161.

BRIEGER, F.G. 1971. Conclusões paleogeográficas e paleoclimáticas baseadas na evolução filogenética e distribuição geográfica de plantas tropicais. Anais da Academia Brasileira de Ciências (Suplemento) 43:197-200.

BRIEGER, F.G. 1976. On the orchid system: general principles and distinction of subfamilies. Proceedings of the $8^{\text {th }}$ World Orchid Conference, p.488-504.

BRYSTING, A.K. \& ELVEN, R. 2000. The Cerastium alpinumC. arcticum complex (Caryophyllaceae): numerical analyses of morphological variation and a taxonomic revision of $C$. arcticum Lange s.l. Taxon 49:189-216.

CARLINI, L.A. 1996. Estudo dos padrões de variabilidade intra e interespecífica em Miltonia Ldl. (Orchidaceae). Dissertação de mestrado, Escola Superior de Agricultura "Luiz de Queiroz", Piracicaba.
DRESSLER, R.L. 1990. The orchids: natural history and classification. Harvard University Press, Cambridge.

DRESSLER, R.L. 1993. Phylogeny and classification of the orchid family. Dioscorides Press, Portland.

DUFRÊNE, M., GATHOYE, J.-L. \& TYTECA, D. 1991. Biostatistical studies on western european Dactylorhiza (Orchidaceae) - D. maculata group. Plant Systematics and Evolution 175:55-72.

DUPUY, D. J., FORD-LLOYD, B.V. \& CRIBB, P.J. 1985. A numerical taxonomic analysis of Cymbidium, section Iridorchids (Orchidaceae). Kew Bulletin 40:421-434.

GARAY, L.A. \& STACY, J.E. 1974. Synopsis of the genus Oncidium. Bradea 1 (40) :393-427.

KNYASEV, M.S., KULIKOV, P.V., KNYASEVA, O.I. \& SEMERIKOV, V. 2000. Interspecific hybridization in northern eurasian Cypripedium: morphometric and genetic evidence of the hybrid origin of $C$. ventricosum. Lindleyana 15:10-20.

LOOS, B.P. 1993. Morphological variation in Lolium (Poaceae) as a measure of species relationships. Plant Systematics and Evolution 188:87-99.

MANLY, B.F.J. 1994. Multivariate statistical methods: a primer. 2 ed. Chapman \& Hall, London.

MCQUEEN, J. \& MCQUEEN, B. 1993. Orchids from Brazil. The Text Publishing Company, Melbourne.

PABST, G.F.J. 1972. Estudos no gênero Oncidium Sw. (Orchidaceae) - IV. Bradea 1 (15) :137-143.

RESENDE, R.M.S. 1991. Aplicação de técnicas de análise multivariada e eletroforese de isoenzimas em estudos de relações fenéticas no gênero Laelia seção Parviflorae. Dissertação de mestrado, Universidade de São Paulo, Piracicaba.

SNEATH, P.H. \& SOKAL, R.R. 1973. Numerical taxonomy: the principles and practice of numerical classification. W.H. Freeman and Company, San Francisco.

VAN DEN BERG, C. 1996. Estudo dos padrões de variabilidade intra e interespecífica em espécies brasileiras de Cattleya Lindley (Orchidaceae-Laeliinae). Dissertação de mestrado, Universidade Estadual de Campinas, Campinas. 